\title{
Interaction of $\alpha$-Synuclein with Negatively Charged Lipid Membranes Monitored by Surface Plasmon Resonance
}

\author{
Katja Pirc, ${ }^{1}$ Vesna Hodnik, ${ }^{1,2}$ Nataša Poklar Ulrih, ${ }^{2}$ Gregor Anderluh ${ }^{1, *}$
}

\author{
National Institute of Chemistry, Hajdrihova 19, SI-1000 Ljubljana, Slovenia \\ 2 Biotechnical Faculty, University of Ljubljana, Jamnikarjeva 101, SI-1000 Ljubljana, Slovenia \\ * Corresponding author's e-mail address: gregor.anderluh@ki.si
}

RECEIVED: April 26, 2016 * REVISED: November 25, 2016 * ACCEPTED: November 28, 2016

\begin{abstract}
Aggregation of presynaptic protein $\alpha$-synuclein is implicated in the development of Parkinson's disease. Interaction of $\alpha$-synuclein with lipid membranes appears to be critical for its physiological and pathological roles. Anionic lipids trigger conformational transition of $\alpha$ synuclein from its natively disordered into an $\alpha$-helical structure. Here we used surface plasmon resonance (SPR) to determine the affinities of $\alpha$-synuclein for the small unilamellar vesicles composed of anionic 1-palmitoyl-2-oleoyl-sn-glycero-3-phospho-L-serine (POPS) or 1,2-dipalmitoyl-sn-glycero-3-phosphoglycerol (DPPG) and neutral 1-palmitoyl-2-oleoyl-sn-glycero-3-phosphocholine (POPC) lipids. $\alpha$-Synuclein bound in a concentration dependent manner to equimolar mixtures of POPC/POPS and POPC/DPPG vesicles. The affinity of $\alpha$-synuclein for POPC/POPS was 3-fold higher than for POPC/DPPG. These results indicate that headgroup charge is not the only factor contributing to $\alpha$-synuclein-membrane association.
\end{abstract}

Keywords: surface plasmon resonance, $\alpha$-synuclein, small unilamellar vesicles, lipid membranes.

\section{INTRODUCTION}

$\boldsymbol{\alpha}$ -SYNUCLEIN is a soluble presynaptic protein. It may exist in aberrant $\beta$-sheet-rich aggregated state, which is causally implicated in the etiology of Parkinson's disease and other synucleinopathies. ${ }^{[1,2]}$ Although $\alpha$-synuclein is abundantly expressed throughout the brain, ${ }^{[3]}$ its precise function and role in key molecular events that lead to progressive neurodegeneration remain elusive. Mounting evidence links both physiological and pathological role(s) of $\alpha$-synuclein with its association with biological membranes. ${ }^{[4-7]}$ While native $\alpha$-synuclein binds to the surface of synaptic vesicles and mediates synaptic plasticity and neurotransmitter release, ${ }^{[4,5]}$ misfolded oligomeric $\alpha$-synuclein species could contribute to neurotoxicity by membrane disruption. ${ }^{[6]}$ However, Parkinson's disease pathology may be associated with a loss or impairment of physiological $\alpha$-synuclein-membrane interaction. ${ }^{[5]}$
Although membrane-binding properties of $\alpha$-synuclein have been extensively studied, there is a lack of consensus regarding $\alpha$-synuclein membrane preferences and its effect on the bilayer structure. ${ }^{[8,9]}$ In aqueous solution and in intracellular environment, $\alpha$-synuclein exists mainly as a disordered and highly dynamic 140-residue monomer. ${ }^{[10]}$ The sequence of $\alpha$-synuclein can be divided into three domains: (i) a positively charged $\mathrm{N}$-terminal region that contains a series of conserved motifs, proposed to enable membrane-induced amphipathic $\alpha$-helix formation, (ii) a hydrophobic central region which is essential for $\beta$-structure formation, and (iii) a negatively charged C-terminus that remains unstructured in both the aggregated and membrane-bound state. ${ }^{[2,8]}$ Depending on membrane physico-chemical properties, several distinct binding modes have been suggested for $\alpha$-synuclein. ${ }^{[11]}$ Nevertheless, the $\mathrm{N}$-terminal $\sim 100$ residues (encompassing $\mathrm{N}$-terminus and central region) undergo coil-helix transition upon 
membrane binding. ${ }^{[12-15]} \alpha$-Synuclein resides on the membrane surface, with some regions immersing deeper into the hydrophobic core of the bilayer. ${ }^{[9,12,13,15]}$ The membrane affinity of $\alpha$-synuclein is highly influenced by bilayer curvature, charge and phase state, chemical composition of the solution (buffer), and lipid:protein ratio. ${ }^{[7-9]} \alpha$-Synuclein may interact better with highly curved surfaces of small unilamellar vesicles (SUVs), ${ }^{[16-18]}$ which resemble the size of synaptic vesicles.[19] Depending on the lipid model system the $\alpha$-helix adopts extended or horseshoe-like conformation. ${ }^{[12,13,15]}$

Extensive data show that $\alpha$-synuclein preferentially binds with lipids that contain negatively charged polar headgroups, such as phosphatidylglycerol (PG) and phosphatidylserine (PS). ${ }^{[16,18,20-24]}$ The interaction is attributed to electrostatic attraction between anionic membrane surface and cationic lysine-rich $\mathrm{N}$-terminus of $\alpha$-synuclein. ${ }^{[14,23]}$ On the contrary, the $\alpha$-synuclein binding with zwitterionic lipids is still unclear, with various reports on strong, weak, or absent association. ${ }^{[7]}$ However, in addition to electrostatic interactions, hydrophobic interactions are important in the association of $\alpha$-synuclein with membranes. ${ }^{[23-26]}$ We have recently shown that the mechanism of $\alpha$-synuclein binding to lipid membranes is primarily dependent on the surface charge density of the lipid bilayer and the phase state of the lipids. ${ }^{[23]} \alpha$-Synuclein associated with SUVs composed of negatively charged 1,2-dipalmitoyl-sn-glycero3-phosphoglycerol (DPPG) in the phase-transition range and in the liquid crystalline state, but not in the gel phase. $\alpha$-Synuclein did not bind to vesicles of the zwitterionic lipid 1-palmitoyl-2-oleoyl-sn-glycero-3-phosphocholine (POPC). ${ }^{[23]}$ Importantly, $\alpha$-synuclein thermally stabilised anionic vesicles, which could have an impact for vesicle fusion with presynaptic membrane. ${ }^{[23]}$

Here we use surface plasmon resonance (SPR) in order to explore $\alpha$-synuclein interaction with lipid membranes in more detail. SPR has become an established approach for studying molecular interactions of proteins with lipid membranes. We found lipid vesicles that remain intact once bound on lipophilic anchors on the L1 sensor chip $^{[27-30]}$ a very convenient approach to study $\alpha$-synucleinmembrane association. We aimed to assess the binding affinities of $\alpha$-synuclein for equimolar mixtures of anionic 1-palmitoyl-2-oleoyl-sn-glycero-3-phospho-L-serine (POPS) or DPPG with neutral POPC.

\section{EXPERIMENTAL}

\section{Materials}

The phospholipids POPC, POPS, DPPG and 1,2-dipalmitoylsn-glycero-3-phosphocholine (DPPC) were from Avanti Polar Lipids (Alabaster, AL, USA) and were used without further purification. Organic solvents were from Merck
KGaA (Darmstadt, Germany) and were of the purest grades available.

Aqueous solutions were prepared in bidistilled water (Millipore, Billerica, MA, USA) and filtered through $0.22 \mu \mathrm{m}$ filters (Sartorius AG, Goettingen, Germany). All of the experiments were performed in $150 \mathrm{mM} \mathrm{NaCl}, 20 \mathrm{mM} \mathrm{HEPES}$, $\mathrm{pH}=7.0$.

\section{$\alpha$-Synuclein Expression and Purification}

Expression and purification of recombinant human wildtype $\alpha$-synuclein was performed as described previously. ${ }^{[31]}$ Final confirmation of the molecular mass and homogeneity of the recombinantly-produced $\alpha$-synuclein was achieved with matrix-assisted laser-desorption/ionisation-mass spectrometry and sodium dodecyl sulfate-polyacrylamide gel electrophoresis.

\section{$\alpha$-Synuclein Preparation}

$\alpha$-Synuclein stock solution was prepared by dissolving the lyophilized protein in $1 \mathrm{mM} \mathrm{NaOH}$ and adjustment to 150 $\mathrm{mM} \mathrm{NaCl}, 20 \mathrm{mM}$ HEPES, $\mathrm{pH}=7.0$. Plausible precipitates were removed by $45 \mathrm{~min}$ centrifugation at $27,000 \mathrm{~g}$. The protein concentrations were determined spectrophotometrically using a Cary Eclipse spectrophotometer (Varian, Mulgrave, Australia), as described previously. ${ }^{[32]}$

\section{Vesicles Preparation}

Small unilamellar vesicles (SUVs) were prepared by sonication as previously described. ${ }^{[23]}$ Briefly, desired lipids dissolved in the appropriate organic solvent were transferred to a round-bottom flask and dried under vacuum using the rotary evaporator for at least $5 \mathrm{~h}$. The warm buffer (150 $\mathrm{mM} \mathrm{NaCl}, 20 \mathrm{mM}$ HEPES, $\mathrm{pH}=7.0$ ) and one-third of teaspoon of glass beads were added and the flask was agitated vigorously on vortex to remove lipids from the walls. The suspension of large multilamellar vesicles was then sonicated on ice for $30 \mathrm{~min}$ with $10 \mathrm{~s}$ on/off cycles at $40 \%$ amplitude. The obtained SUVs were finally incubated for 45 $\mathrm{min}$ at $45^{\circ} \mathrm{C}$ and centrifuged for $5 \mathrm{~min}$ at $6000 \mathrm{rpm}$ to remove possible remaining parts of sonication probe. Lipid vesicles were prepared fresh for each set of experiments.

\section{Surface Plasmon Resonance (SPR)}

The binding analysis was performed using Series $\mathbf{S}$ Sensor chip L1 and Biacore T100 instrument (GE Healthare) basically following established procedures. ${ }^{[29,30]}$ The system was primed twice with the running buffer $(150 \mathrm{mM} \mathrm{NaCl}$, $20 \mathrm{mM}$ HEPES, $0.1 \mathrm{mg} / \mathrm{ml} \mathrm{BSA}, \mathrm{pH}=7.0$ ) and regenerated using two $60 \mathrm{~s}$ pulses of $0.5 \% \mathrm{SDS}$ at $10 \mu \mathrm{l} / \mathrm{min}$. The 1 $\mathrm{mg} / \mathrm{ml} \mathrm{POPC/POPS}$ or POPC/DPPG SUVs were injected for $300 \mathrm{~s}$ at flow rate $2 \mu \mathrm{l} / \mathrm{min}$ over the second flow cell. First flow cell was left empty and served as a reference surface to monitor possible nonspecific binding of the $\alpha$-synuclein 

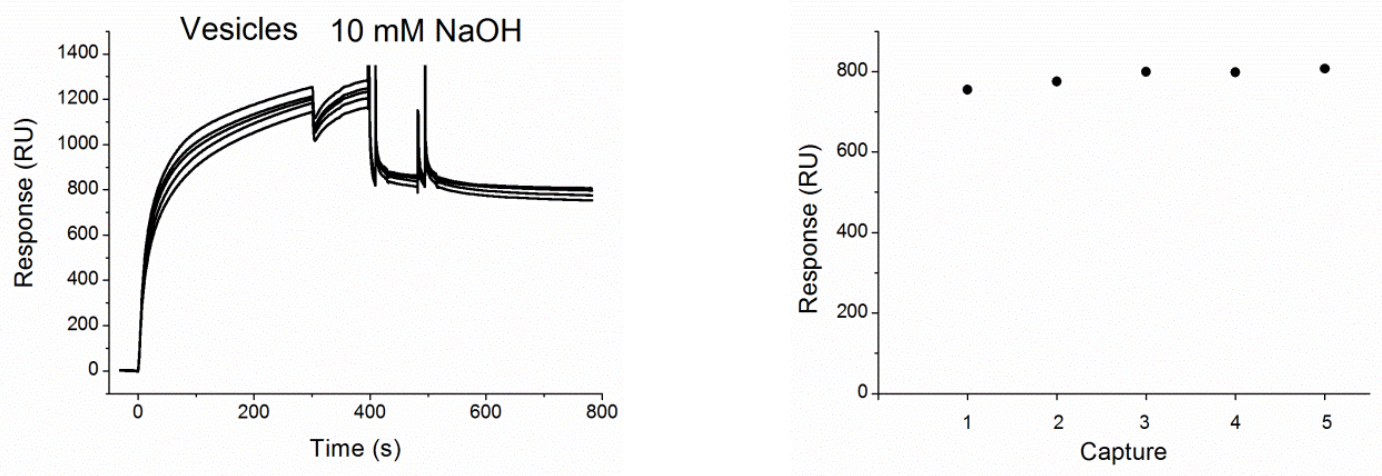

Figure 1. SUV capture. SUVs composed of POPC/POPS or POPC/DPPG were applied to the second flow cell (Fc2) during 5 min injection and were stabilized with 2 short pulses of $10 \mathrm{mM} \mathrm{NaOH}$ (left). The capture level of SUV was kept at the same level (right).

to the surface of the sensor chip. The loosely bound vesicles were washed away with two short pulses (15 s) of $10 \mathrm{mM}$ $\mathrm{NaOH}$ and the surface was allowed to stabilize for 4 minutes, with only buffer running over it, prior each protein injection. The $\alpha$-synuclein was diluted in running buffer $(0$, $2.2,4.4,8.8,17.5$, and $35 \mu \mathrm{M}$ for $\alpha$-synuclein interaction with POPC/POPS and 0, 2.2, 4.4, 8.8, 17.5, 35 and $70 \mu \mathrm{M}$ for $\alpha$-synuclein interaction with POPC/DPPG) and injected over both flow cells for 5 minutes at $10 \mu \mathrm{l} / \mathrm{min}$. Dissociation was monitored for additional 5 minutes. After each protein sample the vesicles were washed away with two 60 s pulses of $0.5 \% \mathrm{SDS}$ at $10 \mu \mathrm{l} / \mathrm{min}$. Stabilization period of $4 \mathrm{~min}$ was introduced prior each subsequent vesicles injection. Experiments were performed at $25^{\circ} \mathrm{C}$.

\section{Data Analysis}

The obtained results were evaluated using Biacore T100 Evaluation software. The sensorgrams were first subtracted by buffer injection (zero concentration) and the reference cell responses and fitted using Steady State Affinity model.

\section{Fluorescence Anisotropy Measurements}

To determine the degree of lipid order of equimolar mixtures of POPC/DPPG and POPC/POPS vesicles, fluorescence anisotropy measurements were performed, as described previously. ${ }^{[23]}$ Briefly, the SUVs were prepared in $150 \mathrm{mM}$ $\mathrm{NaCl}, 20 \mathrm{mM}$ HEPES, $\mathrm{pH}=7.0$ as described above and labelled with 1,6-diphenyl-1,3,5-hexatriene (DPH) fluorescent probe (Sigma-Aldrich Co., St. Louis, MO, USA). For the control, fluorescence anisotropy of 100\% DPPC SUVs was measured. DPH fluorescence anisotropy ( $r$ ) was measured using the built-in software of the Cary Eclipse fluorescence spectrophotometer (Varian, Mulgrave, Australia) at $25^{\circ} \mathrm{C}$, $40{ }^{\circ} \mathrm{C}$ and $60{ }^{\circ} \mathrm{C}$. The excitation wavelength was $358 \mathrm{~nm}$, with the excitation polariser oriented in the vertical position, while the vertical and horizontal components of

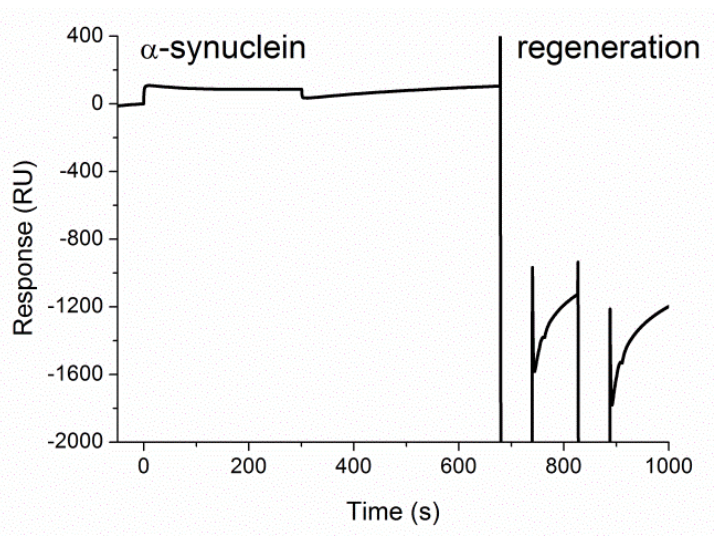

Figure 2. The protein injection. $17.5 \mu \mathrm{M} \alpha$-synuclein was injected over POPC/DPPG for 5 minutes with the 5 minutes of dissociation. The vesicles were removed from the surface with two regeneration steps $(0.5 \% \mathrm{SDS})$ as indicated on the figure.

the polarised emission light were recorded through a monochromator at $410 \mathrm{~nm}$. From the anisotropy value, the lipid order parameter, S, was calculated as previously described. $[23,33]$

\section{RESULTS AND DISCUSSION}

The main benefit of SPR over other biophysical methods is the resolving of the affinity constants from sensorgrams. ${ }^{[30]}$ To assess the interaction of $\alpha$-synuclein with SUVs composed of equimolar mixture of zwitterionic POPC and negatively charged POPS or DPPG, we applied Series $S$ Sensor chip L1. Although $\alpha$-synuclein appears not to interact with $\mathrm{POPC},{ }^{[23]}$ the addition of neutral lipids to anionic can ease vesicle binding onto the sensor chip. The lipid surface was freshly prepared for each $\alpha$-synuclein injection and the capture level was kept similar throughout 

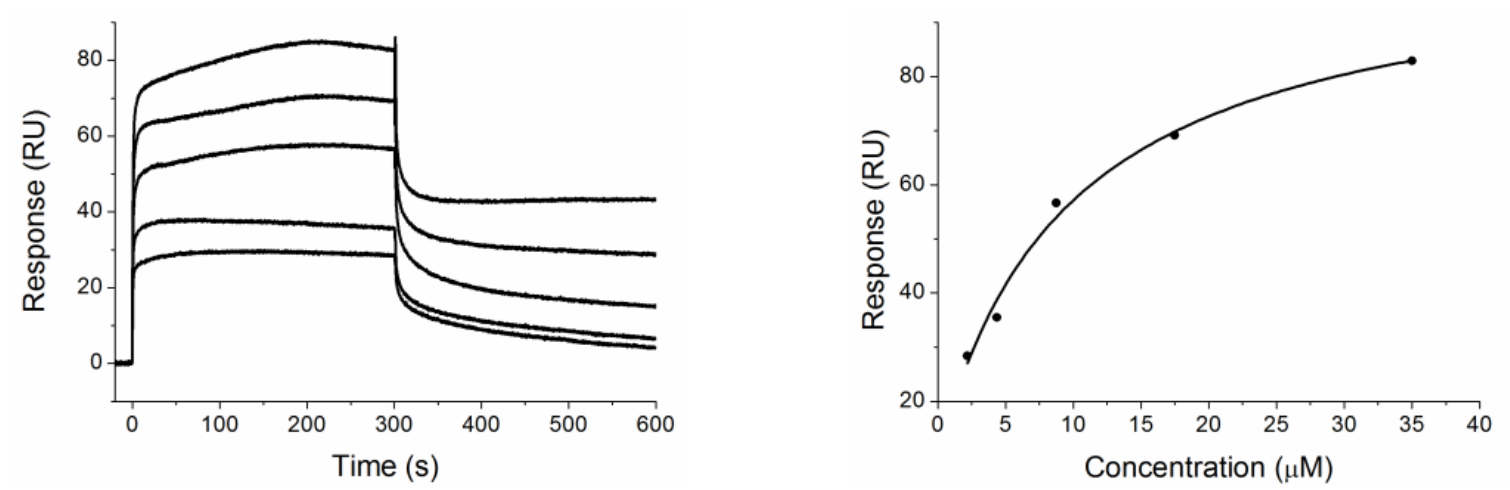

Figure 3. Titration experiments by using POPC/POPS small unilamellar vesicles. $\alpha$-Synuclein $(2.2,4.4,8.8,17.5$ and $35 \mu \mathrm{M})$ was injected over POPC/POPS small unilamellar vesicles (left). The response at the end of the association period (roughly corresponding to the equilibrium response) was plotted against $\alpha$-synuclein concentration in order to obtain an estimate for the affinity constant (right).
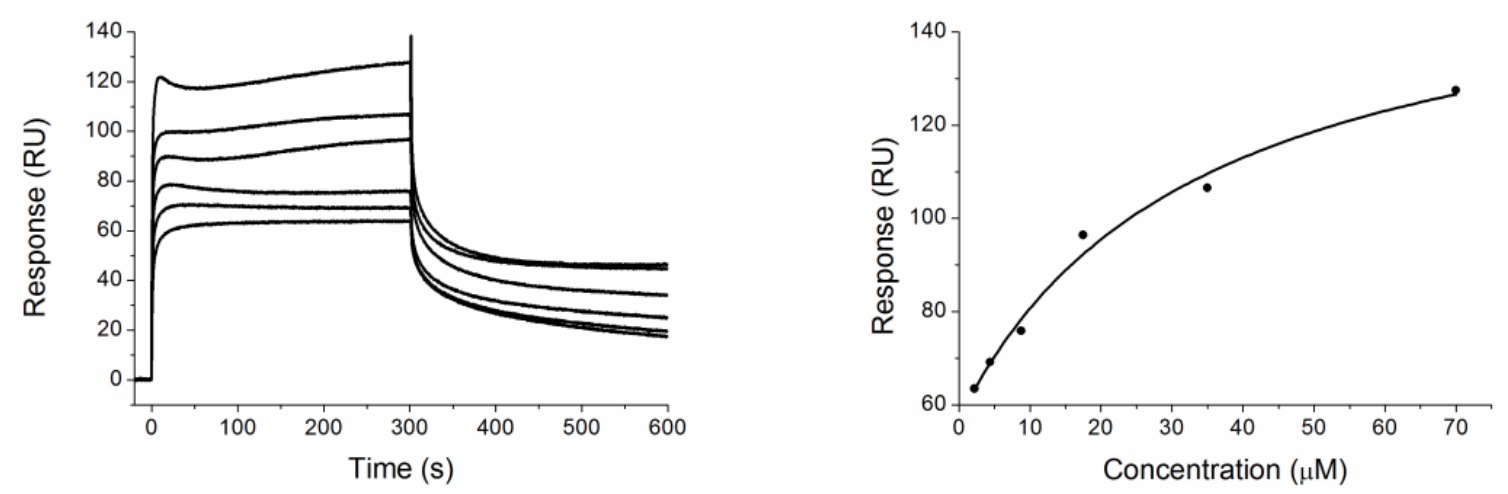

Figure 4. Titration experiments by using POPC/DPPG small unilamellar vesicles. $\alpha$-Synuclein $(2.2,4.4,8.8,17.5,35$ and $70 \mu \mathrm{M})$ was injected over POPC/DPPG small unilamellar vesicles (right). The sensorgrams were evaluated to obtained affinity constant as in Figure 3 (right).

experimentation (approximately $800 \mathrm{RU} ; 787 \pm 21 \mathrm{RU}$ ) for each sample (Figure 1). The SUVs surface was allowed to stabilize for 4 minutes and afterwards $\alpha$-synuclein was injected for 5 minutes with the additional 5 minutes dissociation period. The surface was then regenerated using two $60 \mathrm{~s}$ injection of $0.5 \%$ SDS (Figure 2).

$\alpha$-Synuclein bound in a concentration dependent manner to both, POPC/POPS (Figure 3) and POPC/DPPG vesicles (Figure 4). The injections did not saturate completely the lipid surface within $300 \mathrm{~s}$ of association period indicating the binding is quite complex. Nonetheless we calculated apparent affinity to estimate the binding differences of $\alpha$-synuclein to different lipid headgroups. The affinity of $\alpha$-synuclein for POPC/POPS $\left(K_{D}=9.9 \mu \mathrm{M}\right)$ was approximately 3-fold higher than for POPC/DPPG $\left(K_{\mathrm{D}}=33.5\right.$ $\mu \mathrm{M})$. Also utilizing SPR-based approach Smith et al. reported on apparent dissociation constant of $13.4 \pm 4.6 \mu \mathrm{M}$ for POPC/POPS bilayer, ${ }^{[36]}$ which correlates well with our

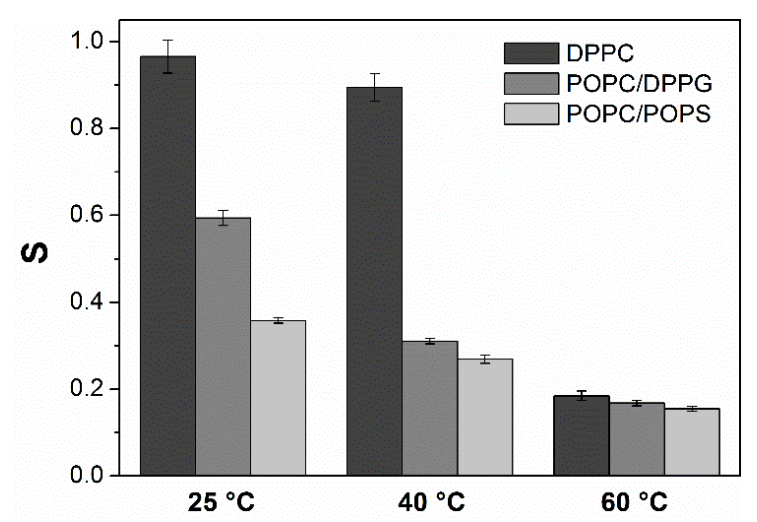

Figure 5. The lipid order parameter, $\mathrm{S}$, of small unilamellar vesicles formed from DPPC, POPC/DPPG and POPC/POPS at $25{ }^{\circ} \mathrm{C}, 40{ }^{\circ} \mathrm{C}$ and $60{ }^{\circ} \mathrm{C}$ (as indicated). Data are means \pm standard error from two independent experiments. Note decrease in S with increasing temperature. 
result. However, caution is needed when directly comparing calculated binding affinities from various studies, as for $\alpha$-synuclein often even minute differences in experimental approach can lead to contradicting results. ${ }^{[7,9]}$ Furthermore, in buffered solution $\alpha$-synuclein exists as an intrinsically disordered monomer, ${ }^{[10]}$ with its conformational heterogeneity extending into higher oligomeric states. ${ }^{[35]}$ For SPR experiment homogenous sample is a prerequisite for satisfactory measurement. Nevertheless, monomeric conformation highly prevails even at the higher $\alpha$-synuclein concentration used here (i.e. $70 \mu \mathrm{M})$. $^{[23]}$ However, $\alpha$ synuclein interaction with lipid vesicles appears to be quite complex. The injections of 17.5 and $35 \mu \mathrm{M} \alpha$-synuclein over POPC/POPS bilayer were affected by dilution with running buffer (Figure 3). Nevertheless, approximately half of the protein remains stably bound on both lipid compositions even after 5 minutes of dissociation period. This indicates that $\alpha$-synuclein does not interact only with the anionic lipid headgroups but rather inserts deeper into the lipid bilayer, as we previously suggested.[23]

Considering that $\alpha$-synuclein does not interact with neutral $P C,{ }^{[23]}$ the binding affinities would suggest preferential $\alpha$-synuclein association with anionic PS over PG headgroup. However, others reported on negligible binding affinity differences for these two anionic headgroups. ${ }^{[18]}$ Indeed, the effect of bilayer fluidity on $\alpha$-synuclein membrane affinity cannot be neglected. We and others have recently shown that $\alpha$-synuclein preferentially binds to anionic bilayers in liquid-crystalline phase, where lipid headgroups and alkyl chains are loosely packed.[23,24] DPPG contains two saturated alkyl chains and at the temperature of the experiments (i.e. $25^{\circ} \mathrm{C}$ ) forms gel phase whereas POPS contains one saturated and one unsaturated alkyl chain and forms liquid-crystalline phase. At temperature of $25^{\circ} \mathrm{C}$ the degree of lipid ordering is lower for the POPC/POPS than for POPC/DPPG vesicles (Figure 5), indicating more fluid phase for the former lipid mixture. Furthermore, the degree of lipid ordering for POPC/POPS is comparable to that of $100 \%$ POPC and POPG, ${ }^{[23]}$ whereas for POPC/DPPG it shows intermediate values between DPPC (gel phase) and POPC/POPS (liquid-crystalline phase) (Figure 5). Therefore, POPC/POPS vesicles might thus provide more accessible hydrophobic region, necessary for $\alpha$-helix stabilization.

Acknowledgment. We dedicate this paper to the memory of prof. Ivana Weygand Đurašević, with whom we successfully collaborated on molecular interaction studies. The work in this paper was supported by program grants P1-0391 and P4-0121 from the Slovenian Research Agency.

\section{REFERENCES}

[1] M. Goedert, M. G. Spillantini, K. Del Tredici, H. Braak, Nat. Rev. Neurol. 2013, 9, 13.

[2] L. Breydo, J. W. Wu, V. N. Uversky, Biochim. Biophys. Acta 2012, 1822, 261.

[3] A. Iwai, E. Masliah, M. Yoshimoto, N. Ge, L. Flanagan, H. A. Rohan de Silva, A. Kittel, T. Saitoh, Neuron 1995, 14, 467.

[4] P. K. Auluck, G. Caraveo, S. Lindquist, Annu. Rev. Cell Dev. Bi. 2010, 26, 211.

[5] H. Lashuel, C. R. Overk, A. Ouslati, E. Masliah, Nat. Rev. Neurosci. 2013, 14, 38.

[6] M. T. Stöckl, N. Zijlstra, V. Subramaniam, Mol. Neurobiol. 2013, 47, 613.

[7] K. Pirc and N. P. Ulrih, Alpha-synuclein interactions with membranes, in: Etiology and physiology of Parkinson's disease (Ed.: A. Qayyum Rana), Intech, Rijeka, 2011, pp. 87-110.

[8] I. Dikyl, D. Eliezer, Biochim. Biophys. Acta, 2012, 1818, 1013.

[9] C. M. Pfefferkorn, F. Heinrich, A. J. Sodt, A. S. Maltsev, R. W. Pastor, J. C. Lee, Biophys. J. 2012, 102, 613.

[10] F. X. Theillet, A. Binolfi, B. Bekei, A. Martorana, H. M. Rose, M. Stuiver, S. Verzini, D. Lorenz, M. van Rossum, D. Goldfarb, P. Selenko, Nature 2016, 530, 45.

[11] C. R. Bodner, C. M. Dobson, A. Bax, J. Mol. Biol. 2009, 390, 775.

[12] T. S. Ulmer, A. Bax, N. B. Cole, R. L. Nussbaum, J. Biol. Chem. 2005, 280, 9595.

[13] C. C. Jao, B. G. Hegde, J. Chen, I. S. Haworth, R. Langen, Proc. Natl. Acad. Sci. U. S. A. 2008, 105, 19666.

[14] T. Bartels, L. S. Ahlstrom, A. Leftin, F. Kamp, C. Haass, M. F. Brown, K. Beyer, Biophys. J. 2010, 99, 2116.

[15] M. Bisaglia, I. Tessari, L. Pinato, M. Bellanda, S. Giraudo, M. Fasano, E. Bergantino, L. Bubacco, S. Mammi, Biochem. 2005, 44, 329.

[16] W. S. Davidson, A. Jonas, D. F. Clayton, J. M. Georges, J. Biol. Chem 1998, 273, 9443.

[17] F. Kamp, K. Beyer, J. Biol. Chem 2006, 281, 9251.

[18] E. R. Middleton, E. Rhoades, Biophys. J. 2010, 99, 2279.

[19] S. Takamori, M. Holt, K. Stenius, E. A. Lemke, M. Grønborg, D. Riedel, H. Urlaub, S. Schenck, B. Brügger, P. Ringler, S. A. Müller, B. Rammner, F. Graüter, J. S. Hub, B. L. De Groot, G. Mieskes, Y. Moriyama, J. Klingauf, H. Grubmüller, J. Heuser, F. Wieland, R. Jahn, Cell 2006, 127, 831.

[20] V. V. Shvadchak, L. J. Falomir-Lockhart, D. A. Yushchenko, T. M. Jovin, J. Biol. Chem 2011, 286, 13023.

[21] M. Stöckl, P. Fischer, E. Wanker, A. Herrmann, J. Mol. Biol. 2008, 375, 1394. 
[22] L. Kjaer, L. Giehm, T. Heimburg, D. Otzen, Biophys. J. 2009, 96, 2857.

[23] K. Pirc, N. P. Ulrih, BBA-Biomembranes 2015, 1848, 2002.

[24] C. Galvagnion, J. W. Brown, M. M. Ouberai, P. Flagmeier, M. Vendruscolo, A. K. Buell, E. Sparr, C. M. Dobson, Proc. Natl. Acad. Sci. U. S. A. 2016, 113, 7065.

[25] N. Lorenzen, L. Lemminger, J. N. Pedersen, S. B. Nielsen, D. E. Otzen, FEBS Lett 2014, 588, 497.

[26] M. Zhu, J. Li, A. L. Fink, J. Biol. Chem. 2003, 278, 40186.

[27] G. Anderluh, M. Beseničar, A. Kladnik, J. H. Lakey, P. Maček, Anal. Biochem. 2005, 344, 43.

[28] M. Beseničar, P. Maček, J. H. Lakey, G. Anderluh, Chem. Phys. Lipids 2006, 141, 169.

[29] V. Hodnik and G. Anderluh, Capture of intact liposomes in Biacore sensor chips for proteinmembrane interaction studies. in: Surface plasmon resonance: methods and protocols, Methods in molecular biology, Vol. 627 (Eds.: N. J. De Mol and M. J. E. Fischer), Springer, New York, 2010, pp. 201-211.

[30] V. Hodnik and G. Anderluh, Surface plasmon resonance for measuring interactions of proteins with lipid membranes in: Lipid-protein interactions: methods and protocols (Ed.: J. Kleinschmidt), Springer protocols, Humana Press, New York, 2013, pp. 23-36.

[31] K. Pirc, M. Škarabot, L. Pogačnik, E. Žerovnik, N. P. Ulrih, Acta Chim. Slov. 2015, 62, 181.

[32] N. P. Ulrih, C. H. Barry, A. L. Fink, BBA- Mol. Basis Dis. 2008, 1782, 581.

[33] H. Pottel, W. Vandermeer, W. Herreman, Biochim. Biophys. Acta 1983, 730, 181.

[34] D. P. Smith, D. J. Tew, A. F. Hill, S. P. Bottomley, C. L. Masters, K. J. Barnham, R. Cappai, Biochem. 2008, 47, 1425.

[35] G. M. Moriarity, M. K. Janowska, L. Kang, J. Baum, FEBS Lett. 2013, 587, 1128. 